\title{
Cambios ambientales y tróficos a través de un análisis a largo plazo del sistema lagunar Chantuto-Panzacola, Chiapas, México
}

\section{Environmental and trophic changes through a long-term analysis of the Chantuto-Panzacola Lagoon System, Chiapas, Mexico}

\author{
Rocío Gómez-Ortega ${ }^{*}$, Guadalupe de la Lanza-Espino ${ }^{2}$, Cristian Tovilla-Hernández ${ }^{1}$,Everardo \\ Barba-Macías ${ }^{3}$, J. Valle-Mora ${ }^{1}$, Ofelia Castañeda-López ${ }^{4}$ y Eduardo Ramos-Santiago ${ }^{5}$
}

\begin{abstract}
RESUMEN
La eutrofización es el proceso de enriquecimiento del agua por nutrientes, que estimula un incremento en la productividad primaria y frecuentemente está relacionado con las proliferaciones de algas nocivas y con las condiciones de hipoxia/anoxia. Este fenómeno, asociado al incremento de la presión antropogénica, se ha convertido en un problema de proporciones globales, por lo que se han realizado diversos esfuerzos para su detección y control. El presente estudio tuvo como objeto analizar la variación espacio-temporal del sistema estuarino Chantuto-Panzacola, tomando en cuenta registros de más de dos décadas anteriores (1990-2009), y su condición fisicoquímica actual (2015-2016). Se aplicaron y desarrollaron índices tróficos y ecológicos a las condiciones tropicales, así como su variación a lo largo del período de análisis. La cuantificación y el análisis de los parámetros fisicoquímicos e índices tróficos determinaron que existen cambios en su patrón anual a partir del 2008, denominados principalmente por los nitratos más nitritos. Sin embargo, en el último año se observó un mayor predominio del amonio. En todos los nutrientes, comparando poco más de 25 años, se obtuvieron concentraciones mayores en los últimos períodos, cuyas concentraciones aumentaron más del $90 \%$ su promedio anual. La aplicación de los índices tróficos corroboró un activo proceso de eutrofización a partir del año 2008, con énfasis en las lagunas de Chantuto, Cerritos y Panzacola, debido al constante aporte de nutrientes provenientes de los ríos adyacentes que contribuyen al aumento de nitrógeno y fósforo.
\end{abstract}

Palabras claves: Eutrofización, lagunas costeras, nutrientes, fisicoquímico, Chiapas.

\begin{abstract}
Eutrophication is the enrichment of a water body with nutrients, thus fostering an increase in primary productivity; it is frequently related to proliferation of harmful algae blooms and hypoxia/anoxia conditions. Eutrophication and increased anthropogenic pressure have become a phenomenon of global proportions; consequently, efforts are currently underway for their detection and control. This paper analyses the spatial and temporal variation of the Chantuto-Panzacola estuarine system, based on records of more than two decades (1990-2009) and its current

\footnotetext{
El Colegio de la Frontera Sur-Unidad Tapachula, Instituto de Biología, UNAM,

Recibido: 24 de julio de 2017

Corregido: 19 de octubre de 2017

Aceptado: 25 de octubre de 2017

DOI: http://dx.doi.org/10.15359/revmar.9-2.4 
physicochemical condition (2015-2016). Trophic and ecological indexes were applied and developed to tropical conditions, as well as their variation over the period of analysis. Quantification and analysis of physicochemical variables and ecological index determined that there have been changes in the annual pattern of nutrients and trophic index since 2008, mainly determined for nitrates and nitrites. However, during the last year a greater predominance of ammonium was observed. Comparing a little over 25 years, higher concentrations were obtained during the last periods in all nutrients, whose annual average concentrations increased more than $90 \%$. Trophic index corroborated an active process of eutrophication since 2008, with an emphasis on the Chantuto, Cerritos and Panzacola lagoons, due to the constant contribution of nutrients from adjacent rivers that contribute to the increase of nitrogen and phosphorus.

Keywords: Eutrophication, coastal lagoons, nutrients, physicochemical, Chiapas.

\section{INTRODUCCIÓN}

Las lagunas costeras son depresiones de la línea de costa, debajo del promedio de la pleamar más alta, con una comunicación marina permanente o efimera y protegidas del mar por un tipo de barrera (Lankford, 1976), unidades de transición entre la tierra y el mar; además, estos ecosistemas están en continuo cambio por el efecto hidrológico del encuentro de dos masas de agua de diferente origen y propiedades fisicoquímicas, como las descargas de agua continental provenientes de los ríos y las intrusiones marinas (Kjerfve, 1994). A causa de que se ubican al final de las cuencas de drenaje, son someras y particularmente susceptibles a la acción del viento sobre la columna de agua y el sedimento, así como a la acumulación constante de aportes terrígenos, en algunos casos con un prolongado tiempo de residencia de los aportes hídricos con sedimentos (Cloern, 1987), y que conducen a la eutrofización. Debido a ello, las lagunas costeras se pueden encontrar sujetas a un proceso natural y antropogénico (cultural) de eutrofización, que consiste en el enriquecimiento de las aguas con nutrientes a un ritmo que no puede ser compensado por la remineralización total de la materia orgánica, de manera que su descomposición tiene como consecuencia la disminución del oxígeno disuelto (De la Lanza-Espino et al. 2012). En las últimas décadas se ha observado que las diversas actividades humanas, como son la quema de combustibles fósiles, la deforestación y el uso de fertilizantes agrícolas, han acelerado dicho proceso. Este enriquecimiento de nutrientes es incorporado a la zona costera por diferentes vías, como son las descargas superficiales directas, el enriquecimiento de aguas subterráneas y la depositación atmosférica (Bricker et al. 2003; Escobedo-Urías, 2010). El efecto inmediato de dicha alteración se manifiesta por lo común con incrementos tanto en la biomasa fitoplanctónica como en la disminución del oxígeno disuelto en el agua (Vollenweider, 1992; Richadson, 1997). Estos factores normalmente afectan la calidad del agua y las tramas tróficas del ecosistema, que a su vez, causan impactos negativos en la pesca, turismo y de salud pública en la comunidad (Cloern, 2001).

Debido a que la respuesta de los ecosistemas costeros al enriquecimiento 
por nutrientes o eutrofización es un proceso continuo y no una condición estática o de estado trófico (Cloern, 2001; Smayda, 2005; Glibert \& Burkholder, 2006), esta situación es aplicable a los sistemas lagunares tropicales, ya que para su evaluación, es necesario desarrollar estudios a largo plazo que permitan determinar cambios en los parámetros fisicoquímicos y sus posibles efectos en los organismos que dependen de estos ecosistemas.

La eutrofización en los cuerpos lagunares es evaluada por indicadores químicos, índices y modelos, tomando en cuenta las condiciones locales ambientales. Sin embargo, la falta de series de tiempo de las concentraciones de nutrientes con suficiente cobertura temporal que conlleve a documentar el proceso de eutrofización dificulta la elaboración de propuestas que evalúen el grado de eutrofización a través de índices tróficos adecuados. Por lo anterior, el objetivo del presente estudio fue determinar la variación espacio-temporal del sistema estuarino Chantuto-Panzacola, tomando en cuenta registros de más de dos décadas anteriores (1990, 1991, 1997, 1999, 2000, 2001, 2002, 2003 у 2008-2009) у su condición fisicoquímica actual (20152016), así como aplicar y desarrollar índices tróficos adecuados a las condiciones tropicales de las lagunas costeras del sur de Chiapas, y determinando si el estado trófico ha cambiado a lo largo de dos décadas (1990-2016).

\section{MATERIALES Y MÉTODOS}

El Estado de Chiapas se encuentra en el Pacífico mexicano, cuenta con
$255.69 \mathrm{~km}$ de cordón litoral y 87000 ha de superficie estuarina. La Reserva de la Biosfera "La Encrucijada" se localiza al sur del Estado, en la región fisiográfica denominada Planicie Costera del Pacífico, geográficamente ubicada entre los $14^{\circ} 43^{\prime}$ y $15^{\circ} 40^{\prime}$ latitud norte y $92^{\circ} 26^{\prime}$ y $93^{\circ} 20^{\prime}$ longitud oeste (Díaz-Ruizet al. 2004). Posee una superficie de 144868 ha, de las cuales 36216 ha corresponden a dos zonas núcleo (La Encrucijada y Palmarcito) y 108651 ha corresponden a la zona de amortiguamiento (Presidencia de la República de México, 1995).

El sistema lagunar-estuarino Chantuto-Panzacola se localiza dentro de la zona núcleo de la Reserva de la Biosfera "La Encrucijada", entre los $15^{\circ} 09^{\prime}$ y $15^{\circ} 17^{\prime}$ de longitud norte y los $15^{\circ} 09^{\prime}$ y $15^{\circ} 17^{\prime}$ de longitud oeste (Fig. 1). Ubicado entre los municipios de Acapetahua y Mapastepec, posee una extensión total de los cuerpos de agua de 18000 ha (Díaz-Ruiz et al. 2004; Gutiérrez-Mendieta et al. 2006). Está conformado por cinco cuerpos de agua: Chantuto, Campón, Teculapa, Cerritos y Panzacola. Tiene una conexión permanente con el mar a través de la Boca San Juan y la Barra del Castaño, recibe la descarga de los ríos San Nicolás, Cacaluta, Cintalapa, Vado Ancho, Despoblado, Huixtla, Cuilapa y Doña María (Díaz-Ruiz et al. 2004), los cuales desembocan en cada una de las cinco lagunas. El clima de la región es del tipo Am (f)w, es decir, cálido-húmedo con abundantes lluvias en verano. El régimen pluvial en la zona está determinado por la humedad 
proveniente del Océano Pacífico. El estiaje está definido entre los meses de noviembre a abril, con menos del $10 \%$ de la tasa anual de precipitación en el período, mientras que las lluvias se presentan entre mayo y octubre. La precipitación anual promedio es de $2310 \mathrm{~mm}$, de los cuales el 95\% del total se distribuye de mayo a octubre. La marea es de tipo mixto semidiurno con una amplitud de $1.25 \mathrm{~m}$ en la Boca de Palmarcito y una hora de bajamar y pleamar casi simultánea para toda la zona (Márquez et al. 2006; GutiérrezMendieta et al. 2006). Existen diferentes ambientes en el sistema lagunar como son: zonas inundables de manglar salobre y de baja salinidad, vegetación emergente y sumergida (Eichhornia crassipes; Mart., Nymphaea ampla; Mart. y Zucc., Pistia stratiotes; L., Salvinia sp.; Cyperus sp.; Typha dominguensis; PERS., Scirpus validus; Vahl.) (Díaz-Ruiz et al. 2004).

La problemática dentro de los sistemas lagunares costeros del Estado de Chiapas se encuentra relacionada con las actividades humanas como la ganadería, la agricultura y la deforestación asociada a estas. La primera ha provocado aparte de la deforestación de la vegetación original, la conversión en pastizales inducidos; la agricultura ha transformado lo que antiguamente eran selvas en campos de cultivo y ha demandado nuevas áreas agrícolas; a causa de esta demanda se realizó el Plan Hidráulico de la Costa de Chiapas que empezó entre 1975 y 1988 (Eguía-Lis, 2012), el cual incluyó la modificación, desvío y rectificación del cauce de varios ríos sobre la planicie, provocando la modificación de 28 ríos entre el 2006 y el 2009, induciendo grandes impactos sobre los cauces medio y bajo y modificando los mantos acuíferos, volumen, batimetría y hábitat de estos (Calva-Benítez et al. 2006; Flores-Verdugo et al. 2007). Asimismo, las modificaciones y rectificaciones en los ríos ocasionan una ruptura del equilibrio en los procesos naturales de acarreo y depósito de sedimentos en los sistemas lagunares. Dicho acarreo provoca el aumento en el aporte de nutrientes provenientes del arrastre directo de los ríos hacia las lagunas, lo que podría llegar a repercutir en problemas de eutrofización y azolvamiento de las lagunas debido al excesivo enriquecimiento de nutrientes y acarreo constante de sedimentos (Flores-Verdugo et al. 2007).

Los cambios en los parámetros fisicoquímicos se evaluaron utilizando una base de datos de los años 1990, 1991, 1997, 1999, 2000, 2001, 2002, 2003 y 2008-2009, asimismo, la caracterización fisicoquímica para el 2015-2016, en todos los años se cubrieron las dos épocas climáticas (lluvias y estiaje), cabe señalar que los muestreos se realizaron en las mismas estaciones (siete) para que pudieran ser comparables (Fig. 1). Se seleccionaron las diferentes zonas tomando en cuenta la influencia de ríos, mar y áreas con diferente vegetación (manglares y vegetación emergente $\mathrm{y}$ sumergida). Debido a lo somero de las lagunas (promedio de $1.15 \mathrm{~m}$ ), en cada estación se tomaron las muestras a medio metro de profundidad. 

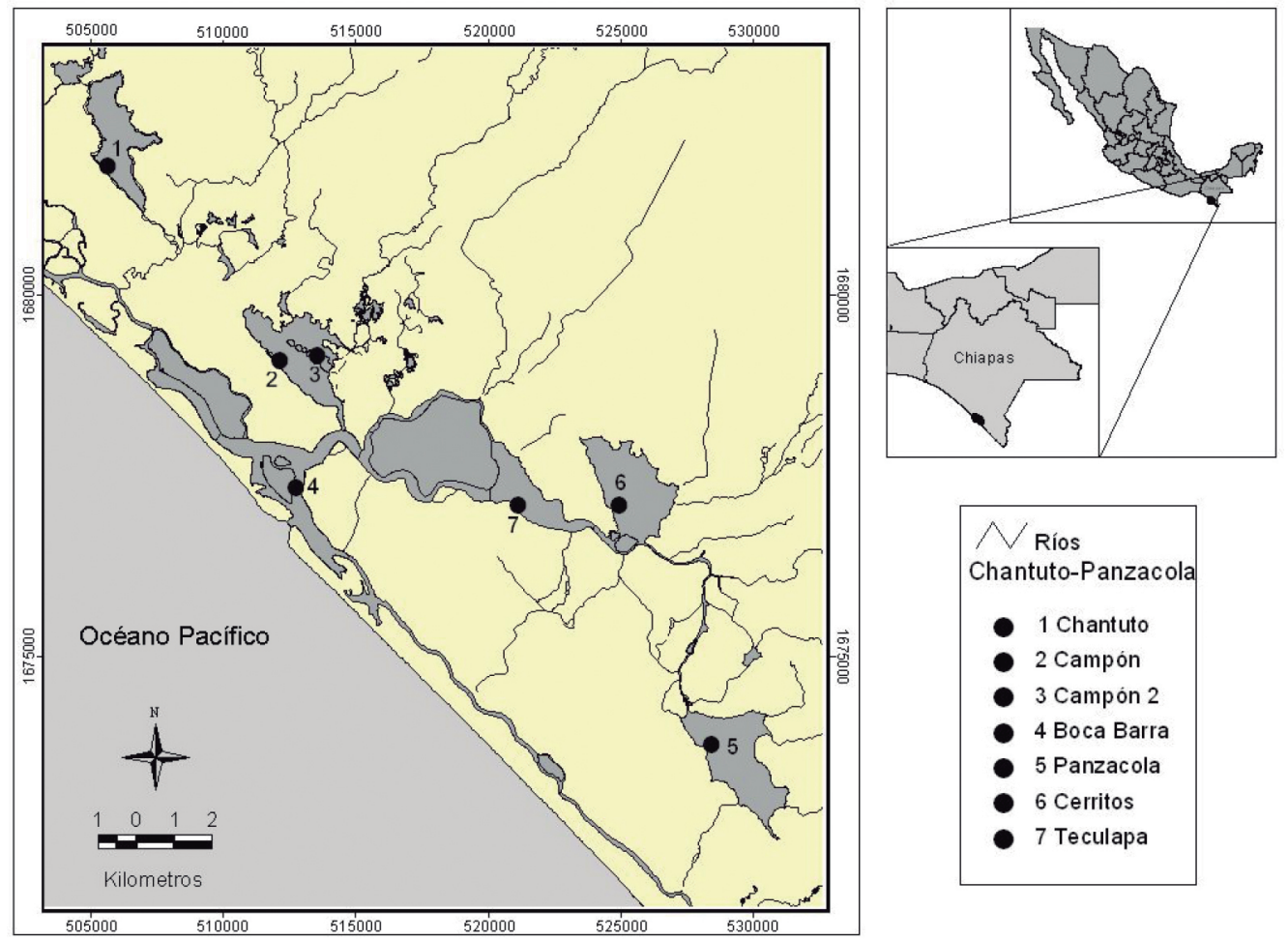

Fig. 1. Sistema lagunar Chantuto-Panzacola, Chiapas, México Fig. 1. Chantuto-Panzacola Lagoon System, Chiapas, Mexico

\section{Procedimiento en campo}

En cada estación se cuantificaron in situ los siguientes parámetros físicos: temperatura $\left({ }^{\circ} \mathrm{C}\right)$ utilizando un termómetro de cubeta, $\mathrm{pH}$ con un potenciómetro Cole-Palmer, salinidad (UPS) con un refractómetro manual óptico y precisión de \pm 1.0 UPS y transparencia y profundidad (m) con el disco de Secchi. El oxígeno disuelto y el porcentaje de saturación se determinaron con una sonda multiparamétrica marca YSI, modelo 85 , que se calibra a nivel de mar cuya precisión es de $\pm 0.3 \mathrm{mg} / 1 \mathrm{y} \pm$ $2 \%$, respectivamente (el oxígeno se corroboró con el método de Winkler).
Para la cuantificación de los nutrientes $\left(\mathrm{NH}_{4}^{+}, \mathrm{NO}_{3}^{-}, \mathrm{NO}_{2}^{-}, \mathrm{PO}_{4}^{-3}, \mathrm{P}\right.$ tot $)$ y $\mathrm{N}$ tot (suma de las formas nitrogenadas), se colectaron muestras de agua empleando una botella van Dorn, estas que se refrigeraron a $4^{\circ} \mathrm{C}$ para su posterior análisis. Todas las muestras se colectaron entre 8 a.m. y 2 p.m.

Para la evaluación de la productividad primaria se utilizó el método de la botella clara y oscura (Gaarder \& Gran, 1927), siguiendo el procedimiento sugerido por Brower y Zar (1981), manteniendo las botellas en incubación en un recipiente de acrílico a bordo de la lancha y cubierto con una malla que reduce la luz alrededor del $20 \%$, con el fin de 
evitar la fotoinhibición de la fotosíntesis. Las muestras fueron incubadas durante tres $\mathrm{h}$, regularmente se efectuaron cambios en el agua para evitar el sobrecalentamiento debido a la insolación.

Para estimar la concentración de clorofila $a$, se filtraron entre 50 y $250 \mathrm{ml}$ de agua a través de filtros de membrana Nucleopore ${ }^{\circledR}$ de 0.45 micras de apertura de poro, agregando un $\mathrm{ml}$ de solución de $\mathrm{MgCO}_{3}$ al $10 \%$, posteriormente, los filtros se desecaron con gel de sílice y se guardaron en tubos de polipropileno de $15 \mathrm{ml} \mathrm{a} 4^{\circ} \mathrm{C}$ para su posterior extracción en el laboratorio.

\section{Procedimiento en laboratorio}

Los nutrientes $\left(\mathrm{NH}_{4}^{+}, \mathrm{NO}_{2}^{-}+\mathrm{NO}_{3}^{-}\right.$, $\mathrm{PO}_{4}^{-3}, \mathrm{PO}_{4}$ ) se analizaron siguiendo las técnicas propuestas por Strickland y Parsons (1972). El equipo utilizado en todas las determinaciones fue un espectrofotómetro marca $\mathrm{HACH}$, modelo DR/2400.

La cuantificación de la clorofila $a$ se realizó mediante la técnica SCORUNESCO (1980), extrayendo con acetona de grado espectrofotométrico al $90 \%$ durante un período mayor a 12 y menor de 24 horas en oscuridad y refrigeración. Las lecturas se realizaron a 665,645 y $630 \mathrm{Nm}$ de longitud de onda, utilizando el multianalizador marca HACH, modelo DR/2400.

\section{Índices ecológicos}

Se determinaron tres índices ecológicos:

Relación N/P (inorgánico, DIN:PO4), se calculó dividiendo las formas de nitrógeno inorgánicas (DIN $=\mathrm{NH}_{4}^{+}, \mathrm{NO}_{3}^{-}, \mathrm{NO}_{2}^{-}$) entre los ortofosfatos ( $\left.\mathrm{PO}_{4}^{-3}\right)$ (Redfield, 1958).
Relación $\mathrm{NH}_{4}^{+} / \mathrm{NT}$, se calculó dividiendo el amonio inorgánico entre la suma de las formas de nitrógeno inorgánicas (Biefang, 1975).

Relación $\mathrm{C} / \mathrm{Cl} \quad a \quad\left(\mathrm{mgCm}^{-3} \mathrm{~h}^{-1}\right.$. mgclo $\left.a m^{-3}\right)$. Esta relación ha sido empleada como índice del potencial fotosintético (Beerman \& Pullingher, 1974), y podría ser considerada como una medida de la eficiencia ecológica por parte del fitoplancton (Banse, 1974; 1977), ya que como puede deducirse, un valor alto significa una optimización de las condiciones abióticas en el desarrollo de los productores primarios; sin embargo, valores bajos estarían relacionados con diversas limitaciones como nutrientes, luz o algún otro factor.

\section{Índices tróficos}

El índice del estado trófico de Carlson (IET), propuesto en 1977, varía entre 0 y 100, es decir, de oligotrófico a hipertrófico. Se obtiene con la transparencia determinada por el disco de Secchi, la concentración de clorofila $a$ y el fósforo total. Sin embargo, la ecuación basada en la cantidad de clorofila $a$ fue la más aceptada. Se utilizó la modificación que realizó Contreras-Espinosa et al. (1994) que plantea una expansión en los intervalos de la concentración del pigmento.

$$
I E T=10\left(2.46+\frac{\operatorname{In}[\text { Clo } a]}{\operatorname{In} 2.5}\right)
$$

El índice de TRIX es un índice multivariado denominado Índice del Estado Trófico TRIX, propuesto por 
Vollenweider et al. (1998), mediante la fórmula:

$T R I X=[\log (C l a *|\% O d| * N I D * P R S)+1.5] / 1.3$

Donde cada uno de los cuatro componentes representa un estado trófico variable:

$\mathrm{Cl} a=$ concentración de clorofila $a \mathrm{mg} / \mathrm{m}^{3}$.

$|\% \mathrm{Od}|=$ valor absoluto de la desviación del por ciento de saturación de oxígeno disuelto, es decir $[100-\% \mathrm{Od}]$.

NID = nitrógeno inorgánico disuelto $\mathrm{N}$ como: $\left[\mathrm{N}-\mathrm{NO}_{3}+\mathrm{N}-\mathrm{NO}_{2}\right.$ $+\mathrm{N}_{-} \mathrm{NH}_{4}$ ], en $\left(\mu \mathrm{g} \mathrm{N} \mathrm{1}^{-1}\right)$

$\mathrm{PO}_{4}=$ ortofosfatos $(\mu \mathrm{g})$.

Las constantes $\mathrm{K}=1.5$ y $\mathrm{m}=$ $12 / 10=1.2$ son valores de escala introducidos para ajustar el valor límite más bajo del índice y la extensión de la escala trófica relacionada de 0 a 10 unidades TRIX.

Índice de TRIX modificado (TRIX ${ }_{\text {CHAN }}$ ) Con la finalidad de ajustar los coeficientes de escala ( $\mathrm{m}$ y $\mathrm{k}$ ) del índice TRIX y hacerlo aplicable al área de estudio, se utilizó una base de datos de 1990 a 2016, generando una ecuación ajustada a las condiciones de los ambientes costeros de Chiapas, la cual fue denominada como TRIX ${ }_{\mathrm{CHAN}}$. Antes de trabajar con la base de datos para la construcción del TRIX CHAN $_{\text {, se }}$ aplicó la transformación utilizando $\log _{10}$, posteriormente se recalcularon los promedios y la desviación estándar. Para determinar los nuevos coeficientes se definieron los rangos para las cuatro variables $(\mathrm{Cl} a, \% \mathrm{OD}$, NID y PID) de los datos corregidos.
Los límites inferiores fueron fijados usando el límite de detección de cada variable (Strickland \& Parsons, 1972), mientras que el máximo se fijó mediante el cálculo hasta obtener un rango de tres unidades logarítmicas en cada variable, lo anterior con la finalidad de darle suficiente amplitud y generar las ecuaciones del TRIX ${ }_{\text {CHAN }}$ de acuerdo con el criterio mencionado por Vollenweider et al. (1998).

El resultado obtenido para el sistema lagunar Chantuto-Panzacola se muestra en el Cuadro 1, los valores obtenidos de k fueron -2.69 (suma de logaritmos mínimos), mientras que el valor del paso trófico fue de 1.2.

De acuerdo con esto, la ecuación del TRIX CHAN $_{\text {: }}$

$T R I X=[\log (C l a *|\% O d| * N I D * P R S)-(-2.69)] / 1.2$

ElTRIX $_{\text {CHAN }}$ representa una variedad de situaciones tróficas, relacionadas con un ambiente costero que va desde condiciones oligotróficas a eutróficas, teniendo en cuenta que valores cercanos a 10 indicaron una fuerte eutrofización, caracterizados por altas concentraciones de $\mathrm{N}, \mathrm{P}$ y $\mathrm{Cl} a$, además de una baja concentración de oxígeno; mientras que los valores cercanos a cero indican aguas con bajo impacto antropogénico. Cabe señalar que trabajar con promedios amortigua la heterogeneidad de las condiciones tróficas de cada estación de muestreo. Para una estimación de estas situaciones se trabajó con datos puntuales por estación de muestreo, así como con promedios anuales.

El índice de eutrofización por nutrientes (KN y KP), según el método 
Cuadro 1. Límites superior e inferior y rango del índice trófico TRIX ${ }_{\text {CHAN }}$

Table 1. Upper and lower limits and trophic index range TRIX CHAN

\begin{tabular}{ccccc}
\hline Parámetro & Log min & Log max & Rango & Paso trófico \\
\hline \multirow{2}{*}{ Cl $\boldsymbol{a}$} & (Lineal) & (Lineal) & Unidades (Log) & Rango/10 \\
& $\mathbf{- 0 . 8 4 5}$ & $\mathbf{2 . 1 5 5}$ & 3 & 0.3 \\
\%OD & 7.00 & 142.8 & & \\
& $\mathbf{- 0 . 6 4 8}$ & $\mathbf{2 . 3 5 2}$ & 3 & 0.3 \\
NID & 4.45 & 224.95 & & \\
& $\mathbf{- 0 . 4 5 3}$ & $\mathbf{2 . 5 4 7}$ & 3 & 0.3 \\
PID & 2.84 & 352.31 & & 0.3 \\
& $\mathbf{- 0 . 7 4 4}$ & $\mathbf{2 . 2 5 6}$ & 3 & $\mathbf{1 . 2}$ \\
\hline
\end{tabular}

de Karydis et al. (1983), se estimó para el nitrógeno inorgánico disuelto

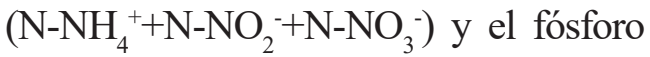
inorgánico $\left(\mathrm{PO}_{4}^{3-}\right)$, basado en la ecuación:

$$
I E=\frac{C}{C-\log X+\log A}
$$

Donde:

$\mathrm{IE}=$ es el índice de eutrofización del nutriente en cada punto (estación) de muestreo durante el período de estudio, compuesto por M muestreos. $\mathrm{C}=$ es el logaritmo de la concentración total del nutriente durante la etapa de estudio en cada punto de muestreo.

$\mathrm{X}=$ es la concentración total del nutriente durante la etapa de estudio en el punto de muestreo.

$\mathrm{A}=$ es el número de puntos de muestreo durante el período de estudio.

Se establece una escala $<3$ para agua oligotrófica, entre 3 y 5 para mesotrófica y $>5$ para eutrófica. Es importante resaltar que este índice se calcula a través de todo el período de análisis, por lo cual se trabajó con promedios anuales por estación de muestreo (laguna) y en su conjunto (por sistema).

\section{Análisis estadísticos}

Se realizó una estimación del $10.11 \%$ de datos faltantes, esto con la finalidad de obtener una matriz válida, de datos completos, ya que durante los muestreos, las condiciones ambientales adversas no favorecieron la toma de datos. La estimación de datos se efectuó empleando el método de imputación de valores, utilizando el paquete missForest, incluido en el programa R (R Core Team, 2016). Por la complejidad de disponer de un total de 7751 datos y 19 variables, se trabajó con valores mínimos y máximos de cada variable por época climática y por año de muestreo, especificando para cada medida el intervalo de 
concentración en el año, además se determinó a qué estación de muestreo pertenecieron. Se decidió analizar los resultados de manera particular debido a la heterogeneidad del sistema, ya que al trabajar con promedios se amortigua esta heterogeneidad, pero aun así, se estimaron los promedios de cada muestreo separados por épocas climáticas. Dado que los datos no cumplieron con los supuestos de normalidad y homocedasticidad, se utilizó un análisis no paramétrico de Kruskal-Wallis en todos los parámetros fisicoquímicos para verificar si existían diferencias significativas entre las medianas de los años de muestreo. Los datos se agruparon en tres conjuntos (D-1: Datos de 1990 a 1999, D-2: Datos del 2000 al 2010, D-3: Datos del 2015 y 2016), con la finalidad de examinar la relación entre los años de estudio y las variables ambientales. Con esta agrupación se realizó un análisis de componentes principales (ACP) que permitió conocer las variables que contribuyen a explicar la mayor variabilidad del sistema. Asimismo, el análisis MANOVA canónico al conjunto de observaciones permitió relacionar dos grupos de datos, por un lado, los parámetros fisicoquímicos (PQ1) y, por el otro, las tres agrupaciones propuestas (D-1, D-2, D-3) (PQ2), lo anterior exploró la correlación entre ambos grupos de variables. Además, se realizó un análisis multivariado de escalamiento multidimensional no métrico, empleándose la métrica de Camberra, con el fin de apreciar las estructuras de agrupación que existen, en este caso se decidió agrupar los datos por los años analizados. Todas las pruebas estadísticas se efectuaron utilizando el paquete estadístico $R$.

\section{RESULTADOS}

\section{Variación anual de los parámetros fisi- coquímicos}

Durante el período analizado la temperatura a media agua presentó su valor mínimo en la estación Chantuto (estiaje) del año 1997 con un intervalo entre $14^{\circ} \mathrm{C}$ y $32^{\circ} \mathrm{C}$, mientras que en la época de lluvias 2008-2009 en la estación Campón2, se determinó el valor más alto cuya variación en ese año fue de $16^{\circ} \mathrm{C}$ a $37^{\circ} \mathrm{C}$. El promedio de la temperatura a media agua por año fue menor en 1997 $\left(28.6^{\circ} \mathrm{C}\right) \mathrm{a} 31.2^{\circ} \mathrm{C}$ en $1999-2001$ (Fig. 2$)$, con diferencias significativas en cuanto a los años de estudio $\left(X^{2}=14.056\right.$, g.l $=$ $5, P=0.01526)$.

La salinidad (UPS) observó un valor mínimo de 0 UPS en la época de lluvias durante la mayor parte de los años muestreados y en todas las estaciones de muestreo, mientras que el valor máximo se presentó en la estación Chantuto durante el estiaje (40 UPS) para los años 1999, 2001, 2008 y 2009. Los promedios anuales de la salinidad por año presentaron el menor de 13.6 UPS en el 2008-2009, mientras que el mayor fue de 20.1 UPS en 1999-2001 (Fig. 2). La salinidad mostró diferencias significativas entre los años de muestreo $\left(X^{2}=28.612\right.$, g.1 $=5, P=2.762 \mathrm{e}-05$ ).

El oxígeno disuelto (OD) mostró una variación semejante entre el 

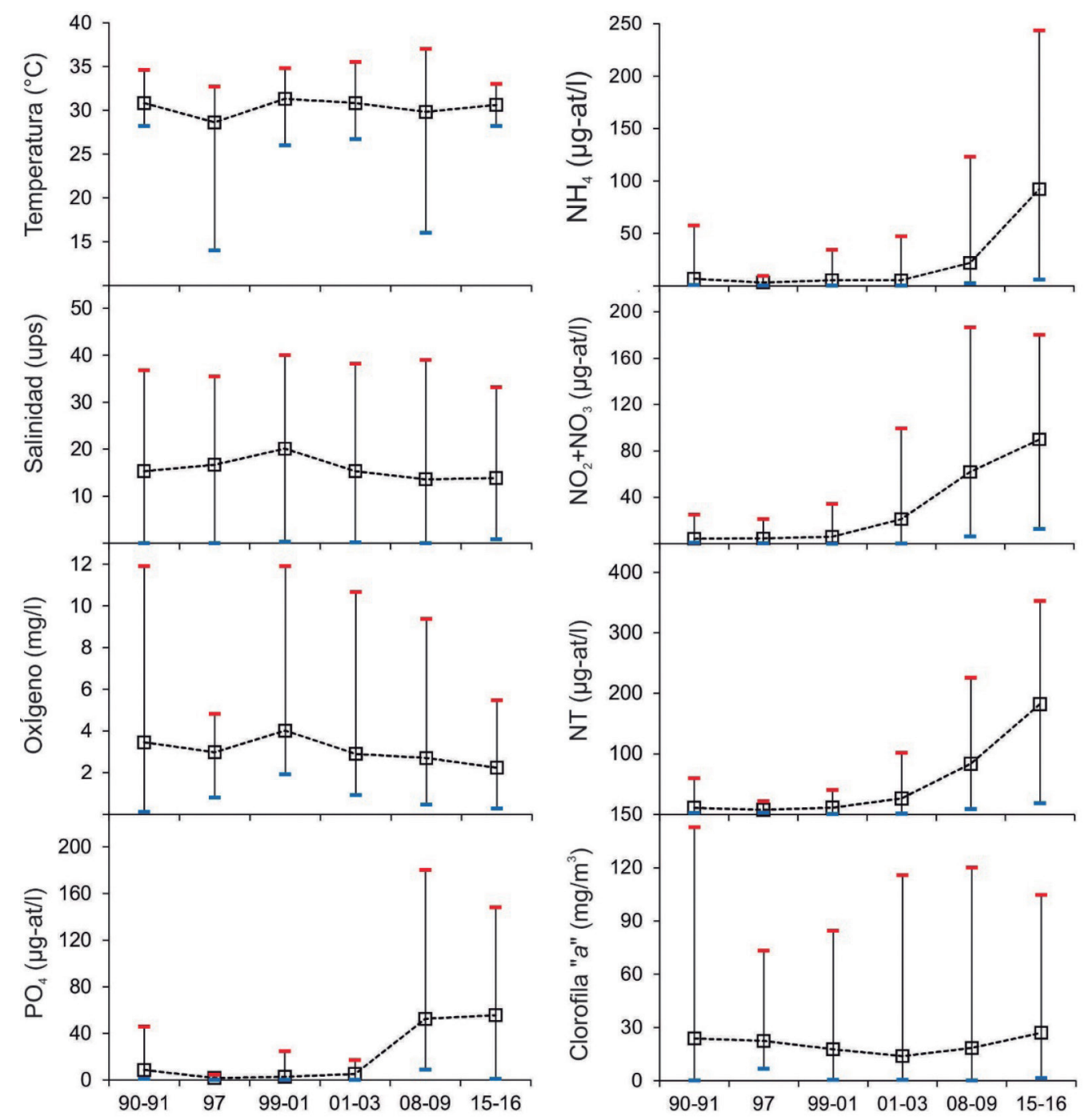

Fig. 2. Máximos, mínimos y promedios de las variables fisicoquímicas y nutrientes por año de muestreo del sistema lagunar Chantuto-Panzacola

Fig. 2. Maximum, minimum and average physicochemical variables and nutrients per sampling year of the Chantuto-Panzacola Lagoon System

estiaje y la época de lluvias de 1990, 1999-2001, de 0.12 a $11.9 \mathrm{ml} / 1$ con diferencias diurnas, siendo este último contenido determinado a medio día bajo condiciones eólicas fuertes. Los promedios anuales mostraron concentraciones ligeramente por arriba de los $2.0 \mathrm{ml} / 1$ en la mayoría de los años analizados (1999, 2001-2003, 20082009 y 2015-2016), mientras que solo en dos períodos las concentraciones estuvieron cercanas a los $4 \mathrm{ml} / 1$ (19901991 y 1999-2001) (Fig. 2). Para determinar las diferencias estadísticas 
de este gas no conservativo, se tomaron todas las concentraciones dentro del ciclo diurno en cuyo caso se registraron diferencias significativas en cuanto a los años analizados $\left(\mathrm{X}^{2}=\right.$ 36.527, g.1 = 5, $P=7.451 \mathrm{e}-07$ ).

La clorofila $a$ exhibió la concentración más baja de todo el período analizado en la estación Boca Barra para el 2008-2009 con un intervalo de 0.09 a $120 \mathrm{mg} / \mathrm{m}^{3}$, semejante a lo registrado en el año 1990-1991 donde se observó la más alta, con un intervalo de 0.09 a $142.8 \mathrm{mg} / \mathrm{m}^{3}$ en la estación Cerritos, ambos valores se presentaron en la época de lluvias. Los promedios anuales registrados oscilaron entre $13.8 \mathrm{mg} / \mathrm{m}^{3}(2001-2003)$ y $27.0 \mathrm{mg} /$ $\mathrm{m}^{3}$ (2015-2016), sin tomar en cuenta la variabilidad espacial (Fig. 2). Si se toman en cuenta todos los registros este parámetro presentó diferencias significativas en cuanto a los años analizados $\left(\mathrm{X}^{2}=31.694\right.$, g. $1=5, P=$ 6.831e-06).

En los años analizados la concentración mínima de Productividad Primaria Neta (PPN) fue nula en 1999-2001-1luvias y estiaje, 2001-2003-lluvias, 2008-2009-1luvias y estiaje, en las estaciones Boca Barra, Campón2, Teculapa y Panzacola, sin embargo, la concentración máxima se registró en el período 1990-1991 con una oscilación de 5.82 a $842 \mathrm{mgC} / \mathrm{m}^{3} / \mathrm{h}$, en la estación Chantuto durante el estiaje. El promedio anual registrado osciló entre $109.7 \mathrm{mgC} / \mathrm{m}^{3} / \mathrm{h}(2008-2009)$ y 161.6 $\mathrm{mgC} / \mathrm{m}^{3} / \mathrm{h}$ (1990-1991), sin discriminar la variación puntual en los años (Fig.
3). La PPN no presentó diferencias significativas en cuanto a los años $\left(\mathrm{X}^{2}=\right.$ 7.4488, g.l = 5, $P=0.1893$ ).

\section{Nutrientes}

La concentración mínima de $\mathrm{NH}_{4}^{+}$ en el período analizado fue en Campón en 1997 (lluvias), con un intervalo de 0.60 a $57.4 \mu \mathrm{M}$, la concentración máxima registrada fue en el estiaje del 2015-2016 en la estación Boca Barra, con un rango de 5.7 a 211.53 $\mu \mathrm{M}$. Los promedios anuales variaron de $3.11 \mu \mathrm{M}(1997)$ a $92.0 \mu \mathrm{M}(2015$ 2016) (Fig. 3); este parámetro presentó diferencias significativas entre años $\left(\mathrm{X}^{2}=183.93\right.$, g. $\left.1=5, P<2.2 \mathrm{e}-16\right)$.

Los niveles de $\mathrm{NO}_{2}^{-}+\mathrm{NO}_{3}^{-}$fueron ampliamente heterogéneos registrando el menor contenido en el período 19992001 durante la época de estiaje, con un intervalo de no detectada (N.D.) en la estación Chantuto a $34.2 \mu \mathrm{M}$, estas concentraciones fueron aumentando hasta alcanzar un máximo en el muestreo del 2008-2009-estiaje en Campón2, presentando una variación de 6.29 a $186.48 \mu \mathrm{M}$, esta se mantuvo durante el año 2015-2016. Los promedios anuales fueron de $4.28 \mu \mathrm{M}$ (1990-1991) a $89.87 \mu \mathrm{M}(2015-2016)$ (Fig. 3). Se observaron diferencias significativas en cuanto a los años analizados $\left(\mathrm{X}^{2}=219.22\right.$, g. $1=5, P<$ 2.2e-16).

La concentración mínima registrada para los ortofosfatos $\left(\mathrm{PO}_{4}^{-3}\right)$ en todo el período analizado fue en 1997, en la estación Chantuto en el estiaje y osciló entre 0.05 a $4.58 \mu \mathrm{M}$, mientras que el máximo registrado fue en el estiaje en 
el 2008-2009, en la estación Campón2, con un intervalo de 8.83 a $180.25 \mu \mathrm{M}$. Presentó promedios anuales de $1.6 \mu \mathrm{M}$ (1997) a $55.6 \mu \mathrm{M}(2015-2016)$ (Fig. 3). Este parámetro mostró diferencias significativas en cuanto a los años $\left(\mathrm{X}^{2}=\right.$ 251.26, g.l = 5, $P<2.2 \mathrm{e}-16$ ).

\section{Índices ecológicos}

El índice $\mathrm{NH}_{4}^{+} / \mathrm{NT}$ presentó valores mínimos en la estación Chantuto en la época de lluvias del 2001-2003, con un intervalo de 0.01 a 0.09 , mientras que el valor máximo de este índice fue registrado en la misma estación en el año 1999-2001 (estiaje), con un rango de 0.07 a 1 , valores cercanos a 1.0 se encontraron durante 1990-1991, 1997, 1999-2001 y 2001-2003 en las estaciones Campón, Campón2, Cerritos, Chantuto y Panzacola. Presentó promedios anuales de 0.27 , el promedio anual menor lo presentó en el 2008-2009 con un valor de 0.27 , mientras que el mayor fue de 0.55 en 1990-1991 (Fig. 3). Este índice mostró diferencias significativas en cuanto a los años de análisis $\left(\mathrm{X}^{2}=\right.$ 66.976, g.l $=5, P=4.358 \mathrm{e}-13$ ).

El índice N/P presentó el valor mínimo y máximo en el estiaje del mismo año (2001-2003) de 0.08 (Teculapa) a 87.11 (Panzacola), con promedios anuales desde 2.46 en el 2008-2009 hasta 9.37 en el 20012003 (Fig. 3). Este índice presentó diferencias significativas $\left(\mathrm{X}^{2}=76.792\right.$, g.l $=5, P=3.93 \mathrm{e}-15)$.

$\mathrm{El}$ índice $\mathrm{C} / \mathrm{Cl} a$ obtuvo valor de cero en los años 1999-2001, 20012003 y 2008-2009 tanto para lluvias, como para el estiaje en la mayoría de las estaciones, excepto Chantuto y Cerritos, con un máximo de 616.9 en el 2001-2003 durante el estiaje en la estación Cerritos. El promedio anual fue de 7.7 en 1997 a 29.49 del 20012003 (Fig. 3). Presentó diferencias significativas en cuanto a los años $\left(\mathrm{X}^{2}=10.919\right.$, g.l $\left.=5, P=0.05302\right)$.

\section{Índices tróficos}

El índice de TRIX CHAN $_{\text {presentó }}$ el valor mínimo durante 1997 en la estación Boca Barra, con un intervalo de 4.03 a 6.96, y un máximo en la estación Chantuto 2008-2009, mostrando una variación de 5.8 a 9.1, ambos valores en el estiaje. Cabe mencionar que en el 2008-2009 y el 2015-2016 se obtuvieron valores por encima de 8.5 , en las estaciones Cerritos (2008-2009-estiaje y lluvias)y Panzacola(2015-2016-lluvias). Los promedios anuales se observaron desde 5.47 (1997) a 7.83 (2015-2016) (Fig. 3), confiriéndoles el nivel trófico de moderado a alto. Este índice presentó diferencias significativas $\left(\mathrm{X}^{2}=231.72\right.$, g.l $1=5, P<2.2 \mathrm{e}-16$ ) en cuanto a los años. La condición trófica que le corresponde por estación de muestreo y año se muestra en el Cuadro 2 y por promedio anual en el Cuadro 3.

El Índice IET $\mathrm{Cl}$ a registró el valor mínimo en Boca Barra del año 2008-2009, cuyo intervalo fue de 0.78 a 76.84 , con un máximo registrado en 1990-1991 en la estación Cerritos, cuya variación fue de 1.16 a 78.74 , ambos valores se presentaron en la época de lluvias. Los promedios anuales variaron de 45.5 (2001-2003) a 57.1 (2015-2016) (Fig. 3), obteniendo 

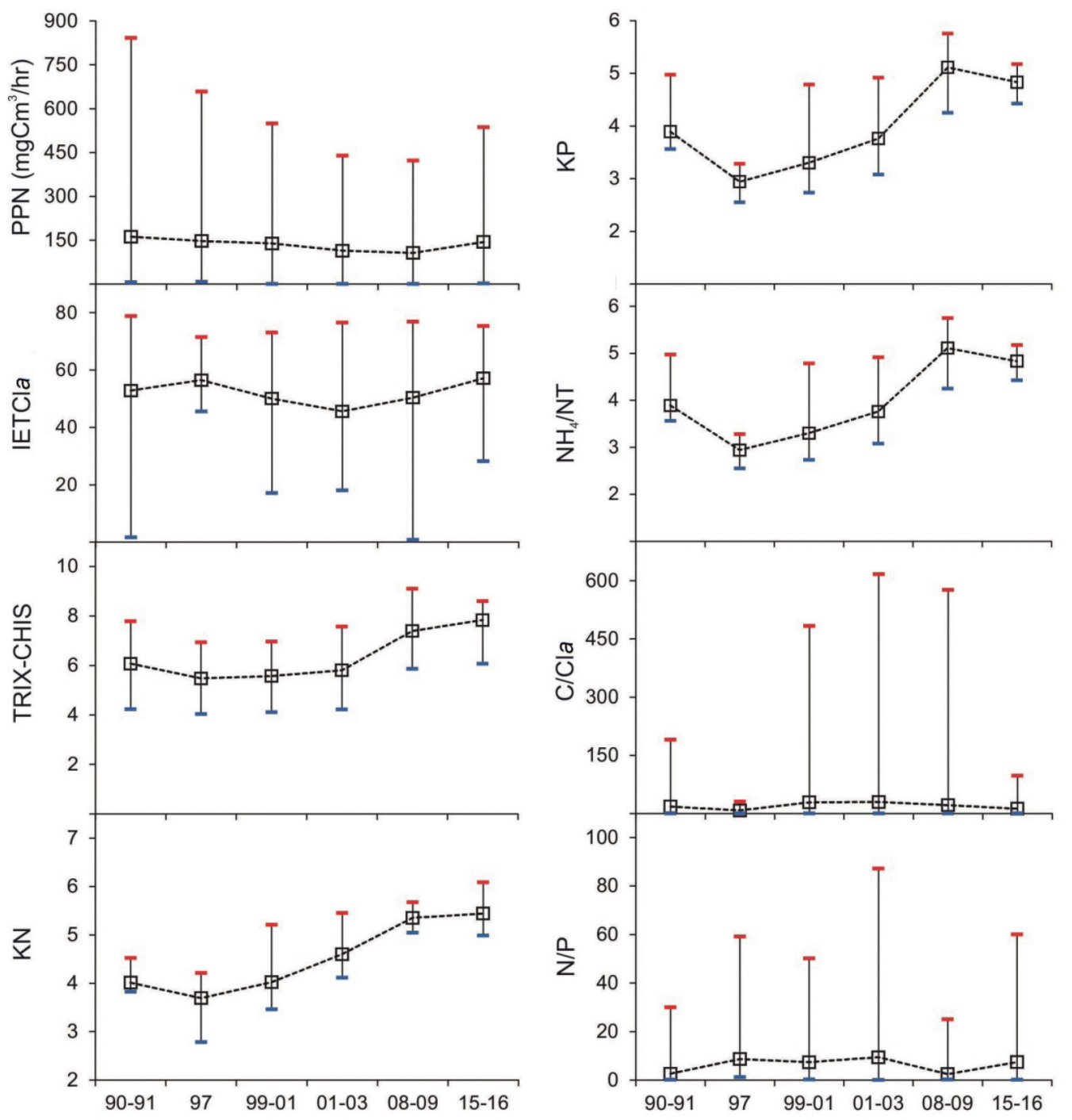

Fig. 3. Máximos, mínimos y promedios de la productividad primaria e índices tróficos por año de muestreo del sistema lagunar Chantuto-Panzacola

Fig. 3. Maximum, minimum and average primary productivity and trophic indices per sampling year of the Chantuto-Panzacola Lagoon System

el nivel trófico de $\alpha$ y $\beta$ eutrófico. Este índice mostró diferencias significativas durante los años de análisis $\left(\mathrm{X}^{2}=\right.$ 31.716, g.1 = 5, $P=6.762 \mathrm{e}-06$ ).

El índice $\mathrm{KN}$ presentó el promedio anual mínimo de 2.78 en 1997 en la estación Boca Barra y el máximo de
5.71 en Campón en el 2015-2016; cabe mencionar que este índice mostró valores por arriba de 5 a partir del año 1999-2001. Los promedios por sistema variaron desde 3.46 (1997) a 5.35 (2008-2009) (Fig. 3). El índice KP mostró promedios anuales de 2.55 en Boca Barra de 1997 


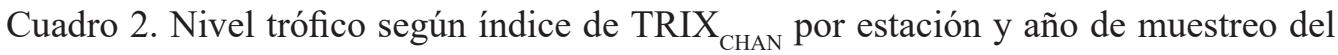
sistema lagunar Chantuto-Panzacola

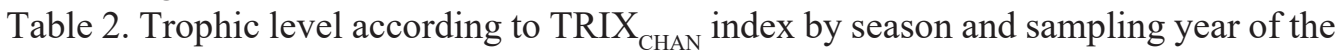
Chantuto-Panzacola Lagoon System

\begin{tabular}{cccccccc}
\hline Estación & $\mathbf{9 0 - 9 1}$ & $\mathbf{9 7}$ & $\mathbf{9 9 - 0 1}$ & $\mathbf{0 1 - 0 3}$ & $\mathbf{0 8 - 0 9}$ & $\mathbf{1 5 - 1 6}$ & Gral. \\
\hline Chantuto & 6.68 & 5.54 & 5.92 & 6.5 & 7.84 & 7.85 & 6.88 \\
Campón & 6.01 & 5.46 & 5.58 & 5.66 & 7.35 & 7.75 & 6.41 \\
Campón2 & 5.94 & 5.54 & 5.02 & 5.63 & 7.04 & 7.77 & 6.25 \\
Boca Barra & 5.6 & 5.12 & 5.53 & 5.7 & 6.85 & 7.56 & 6.16 \\
Panzacola & 5.75 & 5.81 & 5.84 & 5.85 & 7.52 & 7.83 & 6.53 \\
Cerritos & 6.4 & 5.49 & 5.3 & 5.94 & 7.62 & 8.14 & 6.56 \\
Teculapa & 5.97 & 5.37 & 5.74 & 5.5 & 7.48 & 7.86 & 6.43 \\
\hline
\end{tabular}

Cuadro 3. Nivel trófico representado por el índice de $\operatorname{TRIX}_{\text {CHAN }} \mathrm{y}$ el índice de IETCl $a$ del sistema lagunar Chantuto-Panzacola

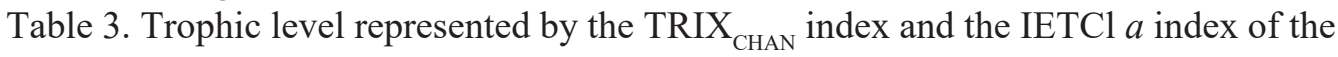
Chantuto-Panzacola Lagoon System

\begin{tabular}{|c|c|c|c|c|c|}
\hline & Época & TRIX $_{\mathrm{CHIS}}$ & Nivel trófico & IETCL $a$ & Nivel trófico \\
\hline \multirow[t]{2}{*}{$90-91$} & Lluvias & 6.21 & Alto & 56.9 & $\beta$-Eutrófico \\
\hline & Estiaje & 5.90 & Moderado & 47.3 & $\alpha$-Eutrófico \\
\hline \multirow[t]{2}{*}{97} & Lluvias & 5.44 & Moderado & 56.8 & $\beta$-Eutrófico \\
\hline & Estiaje & 5.53 & Moderado & 56.0 & $\beta$-Eutrófico \\
\hline \multirow[t]{2}{*}{ 99-01 } & Lluvias & 5.68 & Moderado & 46.5 & $\alpha-$ Eutrófico \\
\hline & Estiaje & 5.46 & Moderado & 53.5 & $\beta$-Eutrófico \\
\hline \multirow[t]{2}{*}{ 01-03 } & Lluvias & 6.10 & Alto & 43.4 & $\alpha$-Eutrófico \\
\hline & Estiaje & 5.49 & Moderado & 47.8 & $\alpha$-Eutrófico \\
\hline \multirow[t]{2}{*}{ 08-09 } & Lluvias & 7.47 & Alto & 48.0 & $\alpha$-Eutrófico \\
\hline & Estiaje & 7.32 & Alto & 54.3 & $\beta$-Eutrófico \\
\hline \multirow[t]{2}{*}{$15-16$} & Lluvias & 7.78 & Alto & 59.7 & $\beta$-Eutrófico \\
\hline & Estiaje & 7.87 & Alto & 54.5 & $\beta$-Eutrófico \\
\hline
\end{tabular}

a 5.75 en Chantuto del 2008-2009. Los promedios por sistema variaron 2079 (1997) a 5.11 (2008-2009) (Fig. 3). Estos índices presentaron diferencias significativas en cuanto a los años de análisis $\left(\mathrm{X}^{2}=232.87\right.$, g.l $=5, P<$
$2.2 \mathrm{e}-16$ y $\mathrm{X}^{2}=243$, g.l $=5, P<2.2 \mathrm{e}-$ 16 , respectivamente). Interpretando, el índice trófico por nutriente mostró una variabilidad de situaciones tróficas, para el caso de KN fue de mesotrófico a eutrófico y para el caso de KP fue 
Cuadro 4. Nivel trófico representado por el índice de KN y el índice de KP del sistema lagunar Chantuto-Panzacola

Table 4. Trophic level represented by the KN index and the KP index of the ChantutoPanzacola Lagoon System

\begin{tabular}{ccccc}
\hline & KN & Nivel trófico & KP & Nivel trófico \\
\hline $\mathbf{9 0 - 9 1}$ & 3.90 & Mesotrófico & 3.77 & Mesotrófico \\
$\mathbf{9 7}$ & 3.46 & Mesotrófico & 2.79 & Oligotrófico \\
$\mathbf{9 9 - 0 1}$ & 4.01 & Mesotrófico & 3.29 & Mesotrófico \\
$\mathbf{0 1 - 0 3}$ & 3.61 & Mesotrófico & 3.43 & Mesotrófico \\
$\mathbf{0 8 - 0 9}$ & 5.35 & Eutrófico & 5.11 & Eutrófico \\
$\mathbf{1 5 - 1 6}$ & 4.01 & Mesotrófico & 3.89 & Mesotrófico \\
\hline
\end{tabular}

desde oligotrófico hasta eutrófico. El correspondiente nivel trófico por año se muestra en el Cuadro 4.

\section{Análisis estadístico}

El análisis de componentes principales (ACP) permitió determinar cuáles son las variables que mayormente contribuyen a explicar la variabilidad del sistema. La Figura 4 representa los parámetros ordenados en tres agrupaciones $(1990,1991,1997,1999=\mathrm{D}-1$; $2000,2001,2002,2003=D-2$ y $2008-$ 2009, 2015-2016=D-3). El análisis de componentes principales explicó el $43.4 \%$ de la variabilidad total presente en los datos, empleando los primeros dos componentes; en el PC1 las variables que tuvieron mayor contribución de forma negativa sobre este componente son: $\mathrm{NO}_{2}^{-}+\mathrm{NO}_{3}^{-}, \mathrm{PO}_{4}^{-3}$, TRIXCHAN, $\mathrm{KN}$ y $\mathrm{KP}$; mientras que el $\mathrm{PC} 2$ estuvo caracterizado de forma negativa por OD, \%Sat, Clor $a$ y el IETCl $a$.

En el análisis de MANOVA canónico se analizaron los parámetros físicos y químicos y los índices ecológicos y tróficos, de las tres agrupaciones de datos. En el análisis se distinguió la D-3 que se separa notablemente de las anteriores, entre los parámetros fisicoquímicos se encontraron cinco parámetros ambientales $\left(\mathrm{PO}_{4}^{3-}, \mathrm{PTot}, \mathrm{NO}_{2}\right.$ $+\mathrm{NO}_{3}^{-}, \mathrm{N}$ Tot y $\mathrm{NH}_{4}^{+}$) y tres índices (TRIX $_{\text {CHIS }}, \mathrm{KN}$ y KP) (Fig. 5).

Utilizando la métrica de Camberra se realizó el Escalamiento Multidimensional no Métrico, donde al estar basado en rangos de distancias se pudo observar una clara transición entre los seis períodos estudiados; observando del lado izquierdo los tres primeros períodos C1, C2 y C3 (1990, 1997 y 1999-2001), mientras que en período C4 fue el de transición (20012003), finalmente separado de los demás períodos, en el lado derecho de la gráfica el C5 y C6 (2008-2009, 20152016) (Fig. 6). El modelo presenta una $\mathrm{R}^{2}=0.95$ y un estrés de 0.24 .

\section{DISCUSIÓN \\ Parámetros fisicoquímicos}

Las lagunas han permanecido de acuerdo con el Sistema de Venecia 


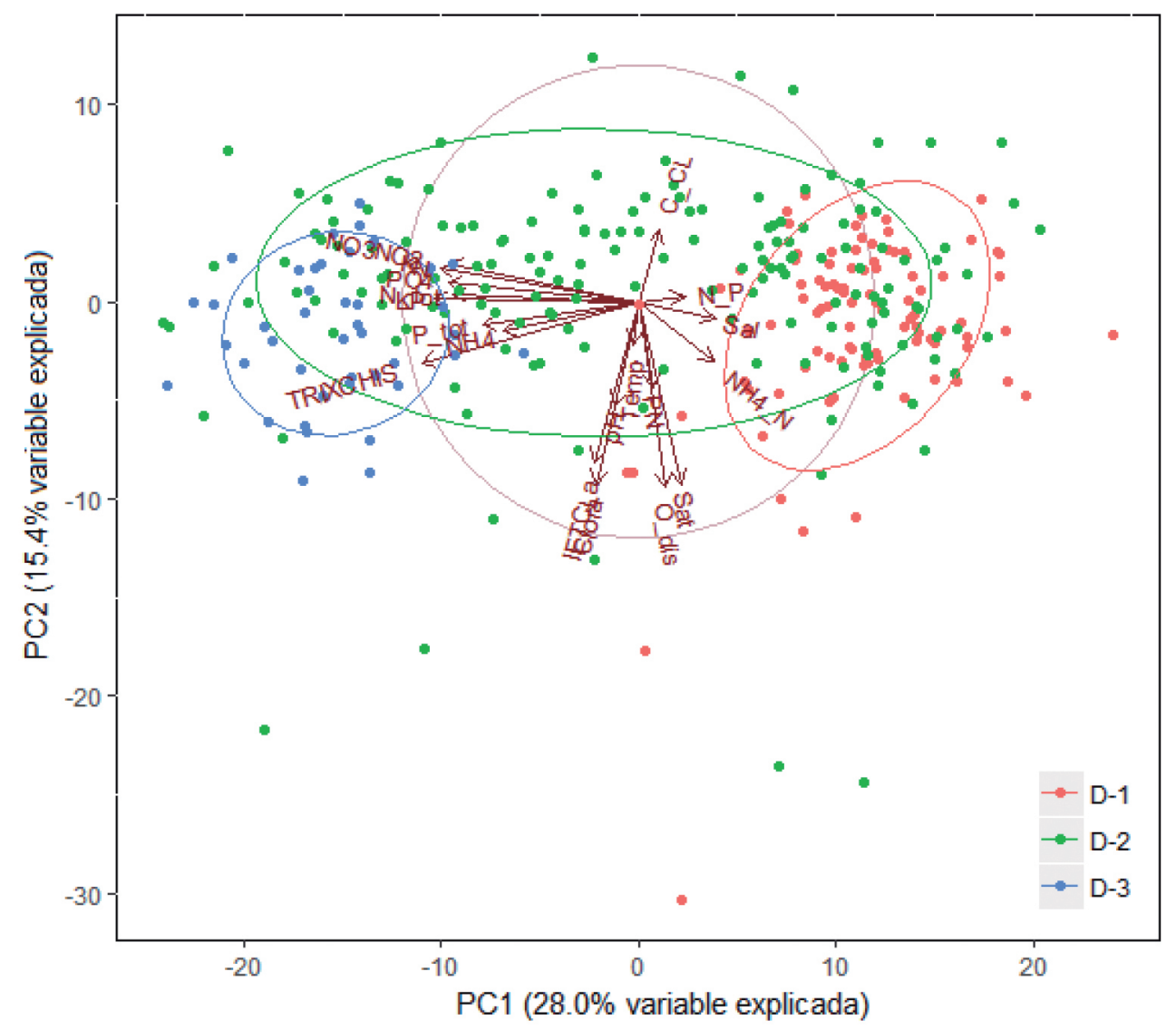

Fig. 4. Componentes principales del sistema lagunar Chantuto-Panzacola

Fig. 4. Principal components of the Chantuto-Panzacola Lagoon System

(1959), como mesohalinas durante los últimos 25 años, con una marcada variabilidad pasando de oligohalinas durante la época de lluvias a euhalinas en el estiaje, indicando con claridad diferencias entre ambas épocas climáticas, influenciadas sobre todo por su geomorfología, la entrada de los ríos y la precipitación pluvial. Cabe mencionar que durante una parte del año, estos sistemas lagunares fueron clasificados como oligohalinos y el resto como estuarino-marinos (García-Nagaya \&
Castañeda-López, 1994). Posteriormente, Contreras-Espinosa (2001) los clasificó como lagunas polihalinas, cuyo promedio anual varió de 18 a 30 ups, mientras que para este estudio y considerando los promedios anuales se clasificaron en mesohalinas. Sin embargo, si se consideran los valores por época climática se observan las fluctuaciones que van desde 0 UPS (oligohalinas) hasta 40 UPS (euhalinas); esta última condición se observó en zonas puntuales donde se tiene una mayor 


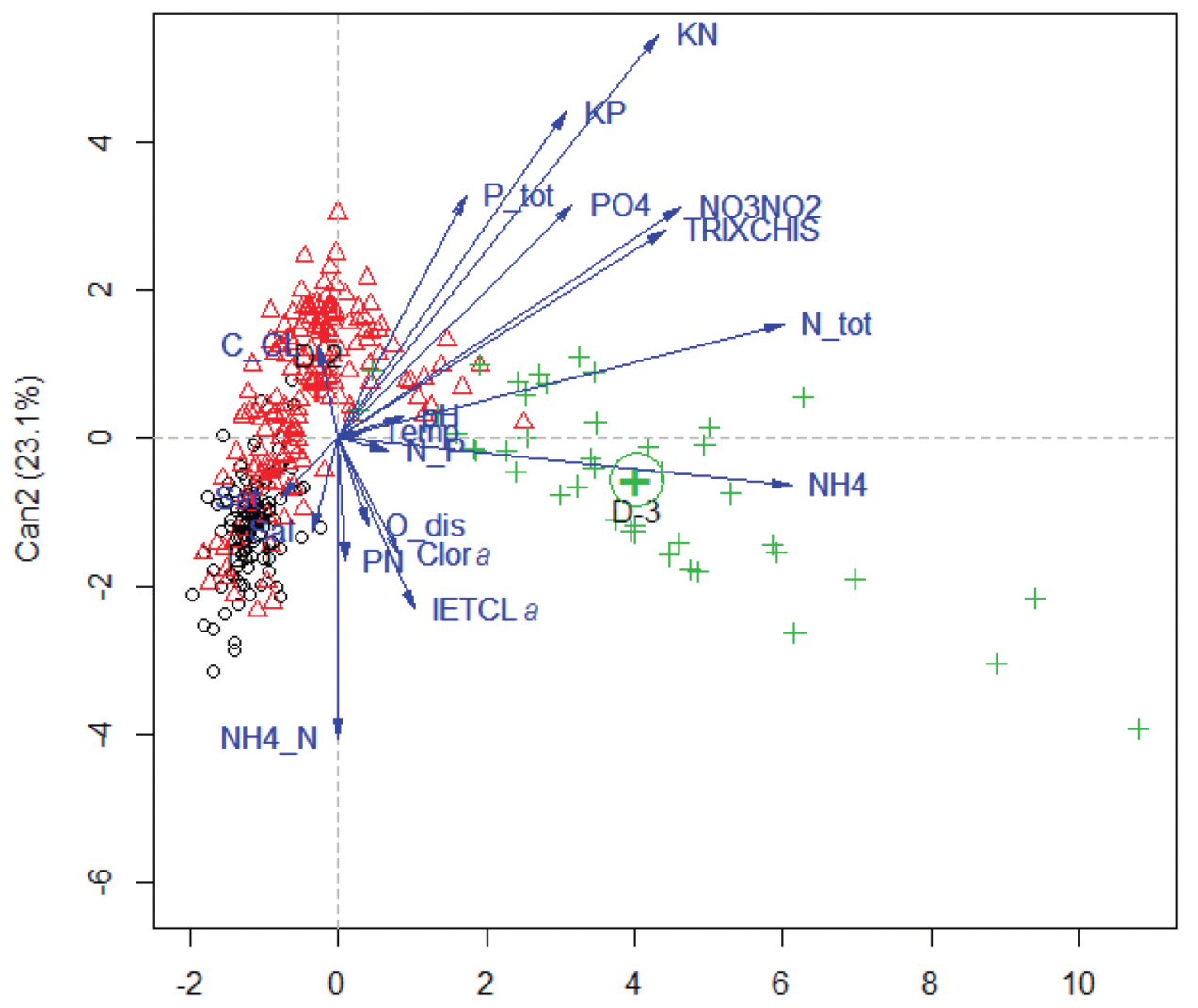

Can1 $(76.9 \%)$

Fig. 5. MANOVA canónica del sistema lagunar Chantuto-Panzacola

Fig. 5. Canonical MANOVA of the Chantuto-Panzacola Lagoon System

pérdida del espejo del agua a causa del azolvamiento de las lagunas, como son el caso de Chantuto, Cerritos y Teculapa, aunado a un incremento en la evaporación. Dicha condición que ha sido descrita por Carbajal-Evaristo (2014) para la laguna Cerritos como resultado del constante arrastre de sedimentos por la canalización del río Cintalapa, pero también como consecuencia de los dragados que se han realizado dentro del sistema (Márquez et al. 2006). Es importante resaltar que, debido a la disminución del espejo de agua, como lo mencionan Gutiérrez-Mendieta et al. (2006), los registros de profundidad del sistema demuestran que se ha perdido parte importante de su volumen, ya que en 1990 se determinó una profundidad promedio de $1.44 \mathrm{~m}$, la cual disminuyó a $1.03 \mathrm{~m}$ en el año 2003. Resalta de manera importante el período 2000-2002, en el que la profundidad promedio fue menor a los 0.90 $\mathrm{m}$, mientras que durante el 2008-2009 el promedio de la profundidad fue de 0.98 m, según Gómez-Ortega (2012). Un análisis a largo plazo indica que 


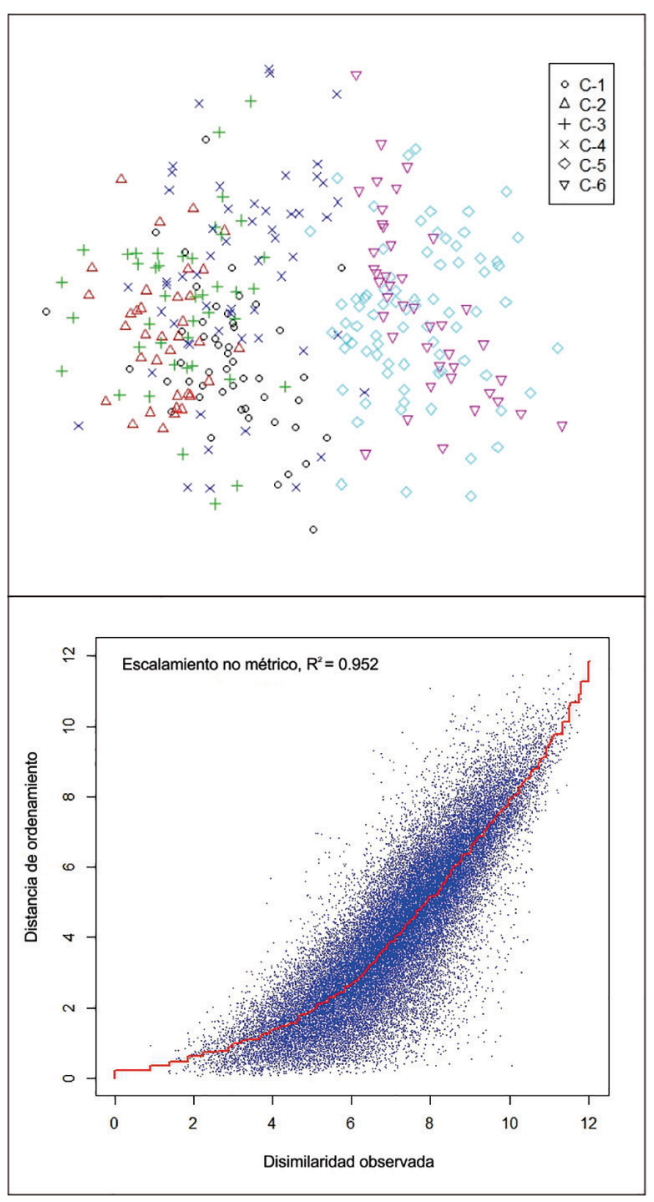

Fig. 6. Escalamiento multidimensional no métrico del sistema lagunar ChantutoPanzacola

Fig. 6. Non-Metric Multidimensional Scaling of the Chantuto-Panzacola Lagoon System

la profundidad aumentó ligeramente a $1.28 \mathrm{~m}$ promedio, como un posible resultado de la interanualidad climática y mareal.

Asimismo, un elemento primordial en los sistemas estuarinos es el oxígeno, componente no conservativo, importante en los procesos de fotosíntesis-respiración y en la descomposición de materia orgánica, también su contenido se considera como un indicador de la salud del sistema (Gürel et al. 2005). Tiene variaciones fuertemente locales como resultado de microclimas, además de estar influenciado por las características geomorfológicas y de circulación (De la Lanza-Espino et al. 2011). Para el sistema lagunar se determinaron bajas concentraciones de este gas, confiriéndole condiciones cercanas a la hipoxia. Al respecto, ContrerasEspinosa (2001) clasificó a estas lagunas como hipóxicas, con valores promedio entre 2 y $3 \mathrm{ml} / 1$, los cuales son considerados como nocivos para la biota acuática. De acuerdo con Gutiérrez-Mendieta et al. (2006) en el 2002 se determinaron niveles de 2.9 $\mathrm{ml} / 1$, y Gómez-Ortega (2012) indicó que para el 2008 los contenidos fueron de $2.7 \mathrm{ml} / 1$. En el presente estudio los valores de oxígeno fueron heterogéneos, oscilando entre lo anóxico, hipóxico e inclusive a la sobresaturación en zonas puntuales relacionadas con el alto contenido de clorofila.

\section{Nutrientes}

La tendencia a largo plazo de los nutrientes $\left(\mathrm{PO}_{4}^{-3}, \mathrm{NO}_{2}^{-}+\mathrm{NO}_{3}^{-} \mathrm{y}\right.$ $\mathrm{NH}_{4}^{+}$) mostró un claro incremento en los promedios anuales con mayores concentraciones a partir del 2008-2009. El amonio es la forma principal para la asimilación de nitrógeno por parte del fitoplancton, y representa el primer compuesto resultante de los procesos de descomposición de los organismos 
muertos y de su metabolismo, por lo que Conway (1977) lo propone como índice de contaminación (ej: la estación de Campón con $211 \mu \mathrm{M}$ ). Este ion aumentó $95.9 \%$ a partir del 2008-2009, y durante el último muestreo su aumento fue mayor a los nitratos más nitritos, lo cual puede interpretarse como una amonificación o degradación de la materia orgánica (proteínas), con la predominancia de amonio sobre los nitritos y nitratos, lo que indicaría mayores tasas de desnitrificación (De la Lanza-Espino et al. 2012). Asimismo, ContrerasEspinosa (2001) propone como concentraciones estándar para lagunas mexicanas, intervalos de 5 a $15 \mu \mathrm{M}$ y comparativamente con el contenido máximo registrado para el sistema lagunar Chantuto-Panzacola este sobrepasa en el 2008-2009, 1.4 veces más $(20.73 \mu \mathrm{M})$, incrementándose durante el 2015-2016 a 6.1 veces $(92.04 \mu \mathrm{M})$ que la propuesta máxima del autor, lo cual indica aportes externos de materia orgánica y un proceso de eutrofización significativo.

El papel de los nitratos y nitritos está determinado por varios factores como son: la fijación del nitrógeno, la asimilación de formas nitrogenadas, la regeneración de nitratos o nitrificación, así como las variaciones estacionales que resultan de la actividad biológica (Contreras, 1983). El intervalo de concentración de estos nutrientes propuesto por Contreras-Espinosa (2001) va desde N.D. (no detectadas) hasta 5 $\mu \mathrm{M}$. En el presente análisis el contenido máximo registrado para el sistema lagunar Chantuto-Panzacola sobrepasó dicho límite a partir del año 1999$2001(6.0 \mu \mathrm{M})$, aumentando 17.9 veces más para el período 2015-2016 (89.8 $\mu \mathrm{M})$, como un posible resultado de las actividades antropogénicas (agricultura, deforestación), dragados, rectificación de los ríos, entre otros factores.

Para el caso del nitrógeno total inorgánico $\left(\mathrm{NO}_{2}^{-}+\mathrm{NO}_{3}^{-}+\mathrm{NH}_{4}^{+}\right)$, Contreras-Espinosa (2001) propone valores de 5 a $20 \mu \mathrm{M}$, concentración que fue acorde hasta el 2001-2003, pero a partir del 2008-2009 aumentó 4.1 veces más $(83.6 \mu \mathrm{M})$ y 9.0 veces más $(181.91 \mu \mathrm{M})$ para el 20152016, de igual manera el incremento está asociado a las actividades antropogénicas.

Los principales aportes de ortofosfatos provienen de ríos que reciben material interperizado o de suelos lixiviados, situación que se corrobora debido a que las máximas concentraciones se registraron en la época de lluvias. Las actividades antropogénicas han provocado un incremento de la concentración de los ortofosfatos en el agua a causa de su baja capacidad de adsorción por parte de los sedimentos, ya que estos colindan con campos agrícolas; y cuyo fenómeno ha sido descrito por De la Lanza-Espino y Arenas-Fuentes (1986) en una laguna del noroeste del Pacífico y Contreras-Espinosa (2001) para el sureste del Pacífico mexicano.

Las concentraciones de ortofosfatos han aumentado de forma parecida a las formas nitrogenadas, donde los incrementos se registraron 
a partir del año 2008-2009 y sobrepasaron las concentraciones máximas propuestas $(5-20 \mu \mathrm{M})$ por Contreras-Espinosa (2001), siendo estas 2.06 veces mayores $(52.4 \mu \mathrm{M})$ en el 2008-2009 y 2.7 veces mayores en el 2015-2016 (55.63 $\mu \mathrm{M})$; lo anterior como resultado de las actividades humanas, tales como cambio del uso de suelo, agricultura, descargas urbanas, entre otras.

De acuerdo con ContrerasEspinosa (2001), los valores normales que se proponen para la PPN de las lagunas costeras es entre 100 y 200 $\mathrm{mgC} / \mathrm{m}^{3} / \mathrm{h}$, concentración que fue rebasada si se analiza de manera puntual (ej: Chantuto-Estiaje 19901991), sin observar tendencia alguna para ciertos años de muestreo.

Asimismo, la $\mathrm{Cl}$ a no mostró ninguna tendencia durante los años analizados, aunque la concentración propuesta fue de $40-50 \mathrm{mg} / \mathrm{m}^{3}$. En el presente estudio solo existieron once datos que cumplieron con esta condición, mientras que 22 datos sobrepasaron estos niveles, y 228 datos fueron menores a la concentración propuesta. Es posible que la concentración de la clorofila $a$ no sea alta y la productividad primaria no aumente aun cuando el contenido de nutrientes haya sido elevado. Este resultado puede mostrar que la respuesta de este indicador biológico al cambio del nutriente no es inmediata, la US-EPA (2001) señala que diferencias entre variables causales (ej: nutrientes) y variables respuestas (ej: clorofila $a$ ) pueden no correlacionarse en determinados sistemas acuáticos. Las razones de lo anterior son múltiples, entre estas destacan el estado de desarrollo de la comunidad fitoplanctónica, el estado fisiológico y la cantidad de feopigmentos o pigmentos accesorios (Margalef, 1974).

\section{Índices ecológicos}

Distinguiendo la heterogeneidad de los datos de manera puntual y con el objeto de asumir si es un valor alto o bajo, para el índice $\mathrm{C} / \mathrm{Cl} a$ se tomó como el promedio general de todos los años analizados el valor de 20.40, siendo así, los valores por encima de este número son considerados como altos y por debajo como bajos; en este entendido existen 64 datos altos y 239 datos bajos, esto se interpretaría según el índice como poco eficiente la clorofila $a$.

Cabe mencionar que una relación $\mathrm{N}: \mathrm{P}$ menor a 5 se interpreta como limitante en nitrógeno, mientras que, mayor a 10 se considera como limitante en fósforo, y de 5 a 10 se establece como indiferente (Rinaldi et al. 1992). En este entendido existieron de manera puntual 206 datos $<5,43$ datos $>10$ y entre 5 a 10 hubo 55 datos, entendiendo que la mayor parte de los datos arrojaron como limitante el nitrógeno.

\section{Índices tróficos}

En el estudio del proceso de eutrofización en ambientes costeros, numerosos métodos se han desarrollado para la evaluación cuantitativa de la eutrofización, tales como técnicas estadísticas, modelos de simulación e indicadores de calidad del agua, siendo 
estos dos últimos los más utilizados para evaluar los niveles tróficos (Karydis, 2009). Los índices o indicadores de eutrofización propuestos tienen la finalidad de lograr la clasificación de las aguas costeras definiéndolas como oligotróficas, mesotróficas o eutróficas, además de resumir una amplia y voluminosa información de campo. Sin embargo, el uso de estos indicadores o índices posee la dificultad de ofrecer una visión fragmentada sobre la eutrofización si son aplicados de forma unitaria, ya que la información puede apuntar a diferencias en el diagnóstico del estado trófico de un sitio al tratar de revisar los resultados. Esta situación se observó en el presente análisis, ya que si consideramos el índice por nutriente (KP), este presentó condiciones que van de oligotróficas (situación que ningún otro índice presentó) a eutróficas, mientras que el $\mathrm{KN}$ exhibió condiciones de mesotróficas a eutróficas. Asimismo, el índice de TRIX CHAN $_{\text {indicó situciones }}$ tróficas de moderadas a altas, y el índice de Carlson presentó de manera puntual una heterogeneidad de situaciones tróficas que van de $\alpha$ a $\beta$-eutrófico, siendo las estaciones Cerritos, Teculapa y Chantuto las que tienen los niveles tróficos más altos.

El análisis estadístico determina que los dos últimos períodos (20082009 y 2015-2016) fueron diferentes de los anteriores y el escalamiento multidimensional no métrico indicó que los años 2001-2003 representan un período de transición. Posterior a este período, se determinó un aumento drástico en los nutrientes, sobre todo en las for- mas nitrogenadas y en menor grado las fosforadas. La presencia de una mayor variabilidad de las condiciones tróficas, principalmente para el NI y el $\mathrm{PO}_{4}^{-3}$ en la época de lluvias, puede sugerir la incorporación de nutrientes a través de los aportes fluviales y de los escurrimientos agrícolas, proceso que ha sido expresado por otros autores (De la Lanza-Espino \& Cáceres, 1994; National Research Council, 2000).

\section{CONCLUSIONES}

Se encontró una tendencia hacia el enriquecimiento principalmente por nutrientes nitrogenados y fosforados a partir del 2008, siendo el amonio la forma de nitrógeno principal en los últimos muestreos, como resultado de la rectificación de ríos, azolvamiento, deforestación, intensificación de la agricultura, cambio del uso del suelo y alteración del paisaje terrestre. Lo anterior sugiere mayores tasas de desnitrificación o una amonificación del sistema, comprometiendo las funciones ecológicas y económicas de estos humedales por el aumento de la concentración de nutrientes y que involucra una serie de respuestas tanto directas como indirectas.

En poco más de 25 años, las mayores concentraciones se observaron en los últimos períodos (2008-2009, 2015-2016), y las formas nitrogenadas aumentaron más del $90 \%$ su promedio anual. Sin embargo, este incremento no mostró correlación con la clorofila $a$ y con la productividad primaria, lo que demostró que la eutrofización fue básicamente por nutrientes. 
La adecuación y aplicación de los índices tróficos de Carlson y TRIXCHAN para los ecosistemas costeros del sur de Chiapas corroboró un activo proceso de eutrofización, sobre todo a partir del año 2008 con énfasis en las lagunas de Cerritos, Teculapa y Chantuto, a consecuencia del constante ingreso de nutrientes.

El empleo de análisis estadísticos con datos a largo plazo permite adaptar herramientas como son los índices tróficos, ya que es posible evaluar y pronosticar tendencias que son importantes para tomar decisiones en políticas públicas y de desarrollo regional; con ello es posible contar con un mayor número de herramientas que conlleven a un manejo eficiente de los ecosistemas costeros y de la conservación de sus recursos naturales.

\section{BIBLIOGRAFÍA}

Banse, K. (1974). On the interpretation of data for the carbonto-Chlorophyll ratio of phytoplankton. Limnol. Oceanogr., 19, 695-699. https://doi.org/10.4319/ lo.1974.19.4.0695

Banse, K. (1977). Determining the Carbon-to-Chlorophyll ratio of natural phytoplankton. Mar. Biol., 41, 199-212. https://doi.org/10.1007/ BF00394907

Beerman, T. \& Pullingher, U. (1974). Annual and seasonal variations of phytoplankton, chlorophyll, and photosynthesis in like Kinneret. Limnol. Oceanogr., 19(1), 31-53. https://doi.org/10.4319/
lo.1974.19.1.0031

Biefang, P. K. (1975). Steady state analysis of nitrate-ammonium assimilation by phytoplankton. Limnol. Oceanogr., 20(3), 402-411. https:// doi.org/10.4319/10.1975.20.3.0402

Bricker, S. B., Ferreira, J. G. \& Simas, T. (2003). An integrated methodology for assessment of estuarine trophic status. Ecol. Model., 169, 39-60. https://doi.org/10.1016/ S0304-3800(03)00199-6

Brower, J. E. \& Zar, J. H. (1981). Field and laboratory methods for general ecology. Iowa, EE.UU.: Wm. C. Brown Company.

Calva-Benítez, L. G., Pérez-Rojas, A. \& Márquez-García, A. Z. (2006). Contenido de carbono orgánico y características textulares de los sedimentos del Sistema Costero Lagunar Chantuto-Pazancola, Chiapas. Hidrobiológica, 16(2), 127-136.

Carbajal-Evaristo, S. (2014). Evaluación del impacto del azolvamiento en La Laguna Cerritos a partir de lla canalización de Río Cintalapa. Tesis de maestría no publicada, El Colegio de la Frontera Sur, Unidad Tapachula, Chiapas, México.

Carlson, R. E. (1977). A trophic state index for lakes. Limnol. Oceanogr., 22, 361-369. https://doi. org/10.4319/1o.1977.22.2.0361

Cloern, J. E. (1987). Turbidity as a control on phytoplankton biomass and productivity in estuaries. Cont. 
Shelf Res., 7, 1367-1381. https://doi. org/10.1016/0278-4343(87)90042-2 Cloern, J. E. (2001). Our evolving conceptual model of the coastal eutrophication problem. Mar. Ecol. Prog. Series., 210, 223-253. https:// doi.org/10.3354/meps210223

Contreras, E. F. (1983). Variación en la hidrología y las concentraciones de nutrientes del área estuarino-lagunar de Tuxpam-Tampamachoco, Veracruz, México. Biótica, 8(2), 201-213.

Contreras-Espinosa, F., CastañedaLópez, O. \& García-Nagaya, A. (1994). La clorofila a como base para un índice trófico en lagunas costeras mexicanas. An. Inst. Cien. Mar. Limnol., 21(1-2), 55-66.

Contreras-Espinosa, F. (2001). Caracterización de lagunas costeras mexicanas a través de variables ecológicas seleccionadas. Tesis de doctorado no publicada, Universidad Autónoma Metropolitana, Ciudad de México, México.

Conway, H. L. (1977). Interactions of inorganic nitrogen in the uptake and assimilation by marine phytoplankton. Mar. Biol., 39, 221-232. https:// doi.org/10.1007/BF00390996

De la Lanza-Espino, G. \& ArenasFuentes, V. (1986). Disponibilidad de nutrimentos a partir de materia orgánica en un sistema lagunar. Ciencia, 37(4), 247-256.

De la Lanza-Espino, G. \& CáceresMartínez, C. (1994). Química de las lagunas costeras y el litoral mexicano. En G. De la Lanza-Espino \&
C. Cáceres-Martínez (Eds.), Lagunas costeras y el litoral mexicano (pp. 127-198). Baja California Sur, México: Universidad Autónoma de Baja California Sur.

De la Lanza-Espino, G., Penié-Rodríguez, I., Czitrom-Baus, S. \& Hernández-Pulido, S. (2011). Química de las lagunas costeras. En G. De la Lanza-Espino \& S. HernándezPulido (Eds.), Ambiente, biología, sociedad y legislación de los sistemas costeros mexicanos (pp. 141177). Distrito Federal, México: Institución de Asistencia Privada y de Plaza Valdés.

De la Lanza-Espino, G., Flores-Baca, B. \& Hernández-Pulido, S. (2012). Laguna de El Yucateco, un ejemplo de recuperación natural. En H. Ramírez-León, J. M. Navarro-Pineda \& H. A. Barrios-Piña (Eds.), Dinámica ambiental de ecosistemas acuáticos costeros (pp. 537-545). Distrito Federal, México: Instituto Politécnico Nacional.

Díaz-Ruiz, S., Cano-Quiroga, E., Aguirre-León, A. \& Ortega-Bernal, R. (2004). Diversidad, abundancia y conjuntos ictiofaunísticos del Sistema Lagunar-Estuarino ChantutoPanzacola, Chiapas, México. Rev. Biol. Trop., 52(1), 187-199. https:// doi.org/10.15517/rbt.v52i1.14879

Eguía-Lis, P. E. (2012). Inventario y valoración del grado de deterioro, cuantificación en campo, así como evaluación, clasificación, delimitación y desarrollo de un programa de manejo integral a nivel de Cuenca 
del Soconusco para la preservación de los humedales presentes en los Ríos Cintalapa, Vado Ancho, Despoblado y Huixtla Región Soconusco, Chiapas. Área 2. Sistema hidrológico Nacional Humedales. México D. F.: Comisión Nacional de Agua.

Escobedo-Urías, D. C. (2010). Diagnóstico y descripción del proceso de eutrofización en lagunas costeras del Norte de Sinaloa. Tesis doctoral no publicada, Instituto Politécnico Nacional, Centro Interdisciplinario de Ciencias Marinas. La Paz BCS, México.

Flores-Verdugo, F. J., Agraz-Hernández, C. \& Benítez-Pardo, D. (2007). Ecosistemas acuáticos costeros: importancia, retos y prioridades para su conservación. En O. Sánchez, M. Herzig, E. Peters, R. Márquez-Huitzil y L. Zambrano (Eds.), Perspectivas sobre la conservación de ecosistemas acuáticos en México (pp. 147-166). México D. F.: Instituto Nacional de Ecología.

Gaarder, T. \& Gran, H. H. (1927). Investigations of the production of plankton in the Oslo Fjord. Rapport. CIEM., 42, 3-31.

García-Nagaya, A. \& Castañeda-López, O. (1994). Hidrología, nutrientes y productividad primaria en dos sistemas costeros del Estado de Chiapas, México. Inv. Mar. CICIMAR., 15(3), 171-188.

Glibert, P. M. \& Burkholder, J. M. (2006). The complex relationships between Increases in fertilization of the earth, Coastal Eutrophication and proliferation of Harmful Algal Blooms. In E. Granéli \& J. T. Turner (Eds.), Ecology of Harmful Algae. Ecological Studies (pp. 341354). Verlag, Berlin: Springer.

Gómez-Ortega, R. (2012). Ecología del Sistema Lagunar ChantutoPanzacola, Chiapas, basada en la aplicación e interpretación de algunos indices tróficos, parámetros físico-químicos y biológicos. Tesis de maestría no publicada, El Colegio de la Frontera Sur, Unidad Tapachula, Chiapas, México.

Gürel, M., Tanik, A., Russo, R. \& Gönec, R. (2005). Chapter. 4 Biogeochemical cycles. In R. Gönec \& J. Wolfin (Eds.), Coastal Lagoons, Ecosystem Processes and modeling for sustainable use and development (pp. 79-192). Boca Raton, Florida, EE.UU.: CRC Press.

Gutiérrez-Mendieta, F., Varona-Cordero, F. \& Contreras-Espinosa, F. (2006). Caracterización estacional de las condiciones físico-químicas $\mathrm{y}$ de productividad primaria fitoplanctónicas de dos lagunas costeras tropicales del estado de Chiapas. Hidrobiológica, 16(2), 137-146.

Karydis, M. (2009). Eutrophication assessment of coastal waters based on indicators: A literature Review. Global NEST Journal, 11(4), 373-390.

Karydis, M., Ignatiades, L. \& Moshopoulou, N. (1983). An index associated with nutrient eutrophication in the marine environmental. 
Estuar. Coast. Shelf Res., 16, 339344. https://doi.org/10.1016/02727714(83)90151-8

Kjerfve, B. (1994). Coastal lagoons. In B. Kjerfve (Ed.), Coastal lagoon processes (pp. 1-8). Amsterdam, Netherland.: Elsevier. https://doi.org/10.1016/ S0422-9894(08)70006-0

Lankford, R. R. (1976). Coastal lagoons of Mexico their origin and classification. In M. Wiley (Ed.), Estuarine Processes (pp. 182-215). Vol II Circulation, sediments, and transfer of material in the estuary. New York, EE.UU.: Academic Press.

Margalef, R. (1974). Ecología. Barcelona, España: Omega.

Márquez, G. A. Z. A., Pérez, R. L. G. \& Calva, B. (2006). Consideraciones batimétricas del sistema lagunar Carretas-Pereyra, Chiapas, México. Hidrobiológica, 16(2), 121-126.

National Research Council. (2000). What determines susceptibility to nutrient over-enrichment? In The National Academies Press (Eds), Clean Coastal Waters: Understanding and Reducing the Effects of Nutrient Pollution (pp. 163-175). Washington, D.C., EE.UU.: National Academies Press.

Presidencia de la República de México. (1995). Decreto por el que se declara como Área Natural Protegida con el carácter de Reserva de la Biósfera, la zona conocida como La Encrucijada. Diario Oficial de la Federación. Recuperado en septiembre 20 de 2017, disponible en http://dof.gob.mx/nota_deta11e.php? codigo $=4875031 \& \mathrm{fec}$ ha $=06 / 06 / 1995$

R Core Team (2016). $R$ : A language and environment for statistical computing. Vienna, Austria: R Foundation for Statistical Computing.

Redfield, A. C. (1958). The biological control of chemical factors in the environment. Amer. Scien., 46, 206-221.

Richardson, K. (1997). Harmful or exceptional phytoplankton blooms in the marine ecosystem. Adv. Mar. Biol., 31, 301$385 . \quad$ https://doi.org/10.1016/ S0065-2881(08)60225-4

Rinaldi, A., Montanari, G., Ghetti, A., Ferrari, C. R. \& Ferrari, A. (1992). Eutrophy and dystrophy in the Goro lagoon. In R. A. Vollenweider, R. Marchetti \& R. Viviani (Eds.), Marine coastal eutrophication (pp. 457-470). New York, EE. UU.: Elsevier Sci.

SCOR-UNESCO. (1980). Determination of chlorophyll in sea water. Technical papers in marine science. In C. J. Lorenzen \& S. W. Jefferey (Eds.), Report of intercalibration tets (pp. 16-17). Cronulla, Australia: United Nations Educational, Scientific and Cultural Organization.

Sistema de Venecia. (1959). Resolución final del Simposio sobre la clasificación de aguas salobres. Archo Oceanogr. Limnol., 11 (supp. 1), 243-248. Smayda, T. J. (2005). Eutrophication and phytoplankton. In P. Wassmann \& K. Olli (Eds.), Drainage Basin 
Nutrient Inputs and Eutrophication: An Integrated Approach (pp. 89-98). Tromso, Norway: University of Tromso.

Strickland, J. \& Parsons, M. R. (1972). A practical handbook of seawater analysis. Ottawa, Canada: Minister of Supply and Services Canada.

US-EPA. (2001). Nutrient Criteria Technical Guidance Manual. Estuarine and Coastal Marine Waters. Washington, D.C., EE.UU.: Environmental Protection Agency.

Vollenweider, R. A., Giovanardi, F., Montarini, G. \& Rinaldi, A. (1998). Characterization of the trophic conditions of marine coastal waters with special reference to the NW Adriatic Sea proposal for a trophic scale, turbidity and generalized water quality index. Environmetrics, 9, 329-357. https://doi.org/10.1002/(SICI)1099095X(199805/06)9:3\%3C329::AIDENV308\%3E3.0.CO;2-9

Vollenweider, R. A. (1992). Coastal marine eutrophication: principles and control. In R. A. Vollenweider, R. Marchetti \& R. Viviani (Eds.), Marine Coastal Eutrophication. Science of the Total Environment (pp. 1-20). Bologna, Italy: Elsevier Science. https://doi.org/10.1016/ B978-0-444-89990-3.50011-0 